\title{
Violência contra idosos: uma análise documental
}

\begin{abstract}
Temática: promoção e prevenção.
Contribuição para a disciplina: este estudo é de grande relevância na construção da ciência de enfermagem e no âmbito da saúde coletiva, uma vez que contribui para a literatura referente à temática da violência contra 0 idoso. Os dados encontrados fomentam a reflexão sobre possíveis influências das relações familiares, questões de gênero, determinantes sociais e de saúde nas diversas expressões da violência. Dessa forma, o conhecimento dos diferentes aspectos que permeiam a violência contra idosos fornece aos profissionais que atuam nos serviços de saúde subsídio para agirem de modo efetivo na prevenção, identificação, enfrentamento e acompanhamento dessas situações. Ademais, esta contribuição facilitará, em longo prazo, a estruturação e aprimoramento de políticas públicas comprometidas com a proteção dos direitos dos idosos.
\end{abstract}

\section{RESUMO}

Objetivo: analisar os casos de violência contra idosos em um município do sul de Minas Gerais (Brasil) num período de 13 anos. Materiais e método: trata-se de um estudo quantitativo, retrospectivo, documental e analítico, que utilizou dados do Conselho Municipal do Idoso. A coleta de dados referentes ao período de 2004 a 2016 ocorreu no primeiro semestre de 2017. Para a análise de tendência dos casos de violência, utilizou-se regressão linear simples. Resultados: foram registradas 389 denúncias, entretanto apenas 273 caracterizavam denúncias de violência contra a pessoa idosa. Quanto ao agressor, 39,56 \% eram do sexo masculino e 43,59 \% eram filhos das vítimas. Com relação ao tipo de violência, destacou-se a negligência $(34,80 \%)$, seguida da violência psicológica $(16,12 \%)$ e financeira $(8,79 \%)$. Evidenciaram-se relação estatisticamente significativa entre sexo da vítima $(p=0,017)$, relação entre vítima e denunciante $(p<0,001)$, e relação entre vítima e agressor $(p<0,001)$ com o tipo de violência. Conclusão: a violência contra 0 idoso nesse município envolve, principalmente, os familiares desses indivíduos; ainda, conclui-se que há necessidade de capacitação dos profissionais e elaboração de uma ferramenta completa e padronizada para o registro de ocorrências e ampliação de investigações na área a fim de se implentarem ações de combate à violência contra o idoso.

PALAVRAS-CHAVE (FONTE: DECS)

Idoso; pessoas de idade; violência; maus-tratos ao idoso; direitos dos idosos; defesa dos idosos; enfermagem.

\section{DOI: 10.5294/aqui.2018.18.4.7}

Para citar este artigo / Para citar este artículo / To reference this article Silva GCN, Almeida VL, Brito TRP, Godinho MLC, Nogueira DA, Chini LT. Violência contra idosos: uma análise documental. Aquichan 2018; 18(4): 449-460. D0I: 10.5294/ aqui.2018.18.4.7

1 orcid .org/0000-0002-4052-981X. Escola de Enfermagem, Universidade Federal de Alfenas, Brasil.

2 orcid.org/0000-0003-0087-5782. Escola de Enfermagem, Universidade Federal do Alfenas, Brasil.

3 orcid.org/0000-0001-9466-2993. Faculdade de Nutrição, Universidade Federal do Alfenas, Brasil. tabatta.brito@unifal-mg.edu.br

4 orcid.org/0000-0003-0826-402X. Escola de Enfermagem, Universidade Federal do Alfenas, Brasil. monica.godinho@unifal-mg.edu.br

5 orcid.org/0000-0003-2285-8764. Departamento de Estatística, Universidade Federal de Alfenas, Brasil.

denismar.nogueira@unifal-mg.edu.br

$6 \triangle$ orcid.org/0000-0003-0266-5295. Escola de Enfermagem da Universidade Federal do Alfenas, Brasil. lucelia.jonas@unifal-mg.edu.br

Recebido: 27/07/2018 Submetido: $22 / 08 / 2018$ Aceito por pares: 19/09/2018 Aceito: 10/10/2018 


\section{Violencia contra los ancianos: un análisis documental}

\section{RESUMEN}

Objetivo: analizar los casos de violencia contra ancianos en un municipio del sur de Minas Gerais (Brasil), en un periodo de 13 años. Materiales y método: estudio cuantitativo, retrospectivo, documental y analítico, que utilizó datos del Consejo Municipal del Anciano. La recolección de los datos referentes al periodo de 2004 a 2016 se dio en el primer semestre de 2017. Para el análisis de tendencia de los casos de violencia, se empleó la regresión lineal simple. Resultados: se registraron 389 denuncios; sin embargo, solo 273 caracterizaban denuncios de violencia contra el adulto mayor. En cuanto al agresor, el 39,56\% era de sexo masculino y el 43,59\% eran hijos de las víctimas. En relación con el tipo de violencia, se destacó la negligencia $(34,80 \%)$, seguida de la violencia psicológica ( $16,12 \%)$ y financiera $(8,79 \%)$. Se evidenció una relación estadísticamente significativa entre el sexo de la víctima $(p=0,017)$, la relación entre víctima y denunciante $(p<0,001)$ y la relación entre víctima y agresor $(p<0,001)$, con el tipo de violencia. Conclusión: la violencia contra el adulto mayor en tal municipio implica, sobre todo, a los familiares de los individuos. Hay necesidad de capacitar a los profesionales que atienden estos casos y hay que elaborar una herramienta completa y estandarizada para el registro de incidencias y ampliación de investigaciones en el área, con el fin de que se implanten acciones que combatan la violencia contra el anciano.

\section{PALABRAS CLAVE (FUENTE: DeCS)}

Anciano; adulto mayor; violencia; maltrato al anciano; derechos de los ancianos; derechos del adulto mayor; enfermería. 


\title{
Violence Against Elderly People: A Documentary Analysis
}

\begin{abstract}
Objective: To analyze the cases of elder abuse in a municipality in the south of Minas Gerais (Brazil) over a 13-year period. Materials and methods: Quantitative, retrospective, documentary, analytical study using data from the Municipal Council of the Elderly. The collection of data for the period between 2004 and 2016 took place in the first half of 2017. For the trend analysis of cases of violence, simple linear regression was used. Results: 389 reports were received, but only 273 described elder abuse. As for the aggressor, $39.56 \%$ were male and $43.59 \%$ were children of the victims. In relation to the type of violence, negligence stood out (34.80\%), followed by psychological $(16.12 \%)$ and financial violence $(8.79 \%)$. There was a statistically significant relationship between the sex of the victim $(p=0.017)$, victim-complainant relationship $(p<0.001)$, victim-aggressor relationship $(p<0.001)$ and the type of violence. Conclusion: Elder abuse in such municipality generally involves relatives of the individuals. It is concluded that there is a need to train professionals and prepare a complete, standardized tool for recording incidents and expanding research in the area in order to take action to fight elder abuse.
\end{abstract}

KEYWORDS (SOURCE: DECS)

Aged; elderly; violence; elder abuse; aged rights; aged advocacy; nursing. 


\section{Introdução}

No contexto do processo de envelhecimento humano, há uma série de alterações físicas, cognitivas, funcionais e sociais que podem levar ao surgimento de morbidades e, por conseguinte, à maior vulnerabilidade a desfechos adversos. Esses fatores somados ao preconceito, ao desrespeito e à desigualdade social podem favorecer a ocorrência de violência contra idosos (1).

De acordo com a Organização Mundial de Saúde (2), a violência contra idosos é "qualquer ação ou omissão (negligência), intencional ou não intencional, que cause dano ou sofrimento às pessoas com mais de 60 anos de idade". Esses abusos podem ser físicos, sexuais, psicológicos ou financeiros.

A violência contra o idoso tem sérias consequências, que incluem sofrimento desnecessário, lesões, dor, medo e podem ocasionar incapacidade, dependência, comprometimento da qualidade de vida do idoso e até mesmo morte $(3,4)$.

Conforme o Plano de Ação para o Enfrentamento da Violência contra a Pessoa Idosa (5), a violência pode ser definida com base na seguinte tipologia: física — refere-se ao uso da força física para obrigar os idosos a fazerem o que não querem, para feri-los, causar-Ihes dor, incapacidade ou morte; psicológica - equivale a agressões verbais ou gestuais com o intuito de amedrontar, humilhar, restringir a liberdade ou isolar a pessoa idosa do contato social; sexual - diz respeito ao ato ou jogos sexuais de natureza homo ou heterorrelacional, que envolvem pessoas idosas no sentido de obter excitação, relação sexual ou práticas eróticas por meio de aliciamento, violência física ou até mesmo ameaças; abandono - trata-se de uma forma de violência que se traduz pela ausência ou abandono dos responsáveis governamentais, institucionais ou familiares, os quais não oferecem socorro à pessoa idosa que necessita de proteção; negligência - consiste na recusa ou omissão de cuidados básicos e essenciais aos idosos, por parte dos responsáveis familiares ou institucionais; financeira — refere-se à exploração indevida/ilegal dos idosos ou ao uso não consentido por eles de seus recursos financeiros e patrimoniais; autonegligência - corresponde à conduta do idoso que ameaça sua própria saúde ou segurança, pela recusa de prover cuidados necessários a si mesmo.

Embora seja um problema de importância social e de saúde pública, trata-se de um tema de difícil investigação, tendo em vista a dificuldade da pessoa idosa em denunciar a própria família, considerada a principal agressora (1). Dados evidenciam que apenas um em cada quatro idosos que sofreram violência registra o caso (6). Cabe ressaltar que estudos que envolvem a temática violência contra o idoso ainda são incipientes, sobretudo em relação à população brasileira, ainda que o entendimento da questão seja fundamental para promover a saúde, o diagnóstico precoce e 0 acompanhamento das vítimas e familiares.

Diante dessa perspectiva, o objetivo deste artigo foi de analisar os requerimentos de denúncia de violência contra idosos registrados no Conselho Municipal do Idoso, em um município do sul de Minas Gerais, Brasil.

\section{Materiais e método}

Estudo quantitativo, retrospectivo, documental e analítico, realizado em um município do sul de Minas Gerais, Brasil, com dados obtidos a partir das denúncias de violência recebidas pelo Conselho Municipal do Idoso. Os dados foram coletados no primeiro semestre de 2017 por alunos de graduação previamente treinados pela coordenadora do estudo.

Consultaram-se os requerimentos de denúncia de violência contra o idoso registrados entre os anos de 2004 e 2016. Nesse período, foram registradas 389 denúncias. Para selecionar os requerimentos, adotaram-se os seguintes critérios de inclusão: requerimentos de denúncia que envolvessem como vítimas pessoas com idade igual ou superior a 60 anos, de ambos os sexos.

Para a coleta dos dados, foi elaborado um roteiro fundamentado nas informações contidas nos requerimentos de denúncia e nas variáveis de interesse dos pesquisadores. 0 roteiro contempla informações referentes à vítima (idade, sexo, estado civil e escolaridade), ao denunciante (idade, sexo e relação com a vítima), ao agressor (idade, sexo e relação com a vítima), ao tipo de violência (física, psicológica, sexual, por tráfico, financeira, por negligência/abandono, por autonegligência, tortura, institucional e outras) e ao desfecho do caso.

Os dados foram codificados no Programa Microsoft Excel $\circledast$ e posteriormente exportados para o software Stata, versão 13.0. 0 estudo evidenciou um gap de informações sociodemográficas, ou seja, registro incompleto nos requerimentos de denúncia sobre os idosos, denunciantes e agressores. As informações não disponíveis nos requerimentos de denúncia foram, dessa forma, consideradas ausentes (não informadas). 
Na análise descritiva dos dados, foram estimadas as proporções. As diferenças entre os grupos foram estimadas por meio do teste Exato de Fisher.

Para a análise de tendência, utilizou-se regressão linear simples. 0 número de casos de violência foi a variável dependente $(\mathrm{Y})$, e os anos de estudo, variável independente $(X)$. Inicialmente, foi construído um diagrama de dispersão entre o número de casos de violência e os anos de estudo, por meio do qual se identificou que a função de primeiro grau seria capaz de expressar a relação de tendência. Foi utilizada a variável $X$ centralizada (ano menos 0 ponto médio da série histórica). Utilizou-se como medida de precisão o coeficiente de determinação $\left(r^{2}\right)$. No modelo, $\beta 0$ representa a média de casos de violência do período analisado e $0 \beta 1$ a velocidade da tendência. Em todas as análises, foi utilizado nível de significância de $5 \%$.

Este estudo obteve a aprovação do Comitê de Ética em Pesquisa da Universidade Federal de Alfenas (CEP/Unifal-MG), sob o parecer de número 1.940.378.

\section{Resultados}

Referente aos dados sociodemográficos (Tabela 1), constatou-se que $37,73 \%$ dos idosos vítimas de violência tinham de 70 a 79 anos, e a maioria era do sexo feminino (58,24 \%). Com relação à situação conjugal e escolaridade das vítimas, a maioria dos requerimentos não apresentava dados sobre essas variáveis. Dentre os denunciantes, observou-se que $18,7 \%$ tinham mais de 60 anos de idade. Destaca-se, entretanto, que 78,02 \% dos requerimentos não possuíam essa informação.

Os denunciantes foram predominantemente do sexo feminino (57,51 \%); além disso, evidenciou-se que os filhos (25,65\%) e os próprios idosos $(21,62 \%)$ denunciavam a maioria dos casos de violência, embora, em 36,26 \% das denúncias, não tenha sido possível constatar essa informação.

Concernentemente às informações do agressor, nos requerimentos dos quais foi possível extrair tais dados, notou-se distribuição semelhante entre adolescentes (3,67\%), adultos entre 20 e 39 anos (5,49\%), adultos entre 40 e 59 anos (5,49\%) e idosos $(5,49 \%)$. Ainda, 39,56\% dos agressores eram do sexo masculino, e 43,59\% eram filhos das vítimas.
Tabela 1. Caracterização das informações do idoso vítima de violência, do denunciante e do agressor no município de Alfenas-MG de acordo com o Conselho Municipal do Idoso, no período de 2004 a 2016 (Alfenas, 2016)

\begin{tabular}{|c|c|}
\hline Variáveis & n (\%) \\
\hline \multicolumn{2}{|c|}{ Variáveis do idoso vítima de violência } \\
\hline \multicolumn{2}{|c|}{ Faixa etária } \\
\hline $60-69$ & $54(19,78)$ \\
\hline $70-79$ & $103(37,73)$ \\
\hline $80-89$ & $75(27,47)$ \\
\hline Mais de 90 & $15(5,49)$ \\
\hline Não informada & $26(9,53)$ \\
\hline \multicolumn{2}{|c|}{ Sexo } \\
\hline Feminino & $159(58,24)$ \\
\hline Masculino & $114(41,76)$ \\
\hline \multicolumn{2}{|c|}{ Estado civil } \\
\hline Solteiro & $1(0,36)$ \\
\hline Casado & $54(19,78)$ \\
\hline Divorciado & $1(0,36)$ \\
\hline Viúvo & $4(1,46)$ \\
\hline Não informado & $213(78,2)$ \\
\hline \multicolumn{2}{|c|}{ Escolaridade } \\
\hline Ensino superior & $1(0,36)$ \\
\hline Não informada & $272(99,64)$ \\
\hline \multicolumn{2}{|c|}{ Variáveis do denunciante } \\
\hline \multicolumn{2}{|c|}{ Faixa etária } \\
\hline 10-19 & $1(0,36)$ \\
\hline $20-39$ & $4(1,46)$ \\
\hline $40-59$ & $4(1,46)$ \\
\hline Mais de 60 anos & $51(18,7)$ \\
\hline Não informada & $213(78,02)$ \\
\hline \multicolumn{2}{|c|}{ Sexo } \\
\hline Feminino & $157(57,51)$ \\
\hline Masculino & $69(25,27)$ \\
\hline Não informado & $47(17,22)$ \\
\hline
\end{tabular}




\begin{tabular}{|c|c|}
\hline \multicolumn{2}{|c|}{ Relação do denunciante com a vítima } \\
\hline Própria vítima & $59(21,62)$ \\
\hline Filhos & $70(25,65)$ \\
\hline Netos & $5(1,83)$ \\
\hline Irmãos & $3(1,09)$ \\
\hline Vizinhos & $4(1,46)$ \\
\hline Outros familiares/Outros & $33(12,09)$ \\
\hline Não informada & $99(36,26)$ \\
\hline \multicolumn{2}{|c|}{ Variáveis do agressor } \\
\hline \multicolumn{2}{|c|}{ Faixa etária } \\
\hline 10-19 & $10(3,67)$ \\
\hline $20-39$ & $15(5,49)$ \\
\hline 40-59 & $15(5,49)$ \\
\hline Mais de 60 anos & $15(5,49)$ \\
\hline Não informada & $218(79,86)$ \\
\hline \multicolumn{2}{|c|}{ Sexo } \\
\hline Feminino & $77(28,20)$ \\
\hline Masculino & $108(39,56)$ \\
\hline Não informado & $88(32,24)$ \\
\hline Relação do agressor com a vítima \\
\hline Filhos & $119(43,59)$ \\
\hline Cônjuge & $7(2,56)$ \\
\hline Outros familiares/Outros & $77(28,2)$ \\
\hline Própria vítima & $15(5,49)$ \\
\hline Não informada & $55(20,16)$ \\
\hline
\end{tabular}

Fonte: elaboração própria.

Dentre os anos de 2004 a 2016, foram registradas 389 denúncias de violência contra a pessoa idosa no Conselho Municipal do Idoso. Dessas notificações, $116(29,8 \%)$ não eram caracterizadas como denúncia de violência contra a pessoa idosa, mas sim se referiam à solicitação de consultas médicas e cirurgias, medicamentos, fraldas, cestas básicas. 0 maior registro de denúncia ocorreu no ano de 2016 (21,6\%). Cabe destacar que alguns requerimentos de denúncia do ano de 2014 não foram localizados no Conselho Municipal do Idoso (Tabela 2).

Observou-se que, dentre os idosos que sofreram somente um tipo de violência, destacou-se a negligência $(34,80 \%)$, seguida da violência psicológica $(16,12 \%)$ e financeira $(8,79 \%)$. Não foram registradas denúncias de violência sexual, tortura ou tráfico. Quanto ao encaminhamento das vítimas, 41,39 \% dos casos de violência eram direcionados ao Centro de Referência Especializado de Assistência Social (Creas), entretanto, em 53,48 \% dos requerimentos avaliados, não havia tal informação (Tabela 2).

Tabela 2. Distribuição dos casos de violência segundo ano, categoria de violência e encaminhamento da denúncia de acordo com o Conselho Municipal do Idoso de Alfenas-MG, no período de 2004 a 2016 (Alfenas, 2016)

\begin{tabular}{|c|c|}
\hline Variáveis & n (\%) \\
\hline \multicolumn{2}{|l|}{ Ano } \\
\hline 2004 & $2(0,73)$ \\
\hline 2007 & $1(0,36)$ \\
\hline 2008 & $20(7,33)$ \\
\hline 2010 & $24(8,80)$ \\
\hline 2011 & $42(15,38)$ \\
\hline 2012 & $40(14,65)$ \\
\hline 2013 & $40(14,65)$ \\
\hline 2014 & $1(0,36)$ \\
\hline 2015 & $44(16,12)$ \\
\hline 2016 & $59(21,62)$ \\
\hline \multicolumn{2}{|l|}{ Tipo de violência } \\
\hline Físico & $14(5,12)$ \\
\hline Psicológico & $44(16,12)$ \\
\hline Financeiro & $24(8,79)$ \\
\hline Abandono/Negligência & $95(34,80)$ \\
\hline Autoabandono/Autonegligência & $21(7,69)$ \\
\hline Institucional & $5(1,83)$ \\
\hline Mais de um tipo de violência & $70(25,64)$ \\
\hline \multicolumn{2}{|c|}{ Encaminhamentos/desfecho dos casos } \\
\hline $\begin{array}{l}\text { Creas/Centro de Referência de Assistência Social } \\
\text { (Cras) }\end{array}$ & $113(41,39)$ \\
\hline Promotoria & $2(0,73)$ \\
\hline Polícia & $1(0,36)$ \\
\hline Mais de um encaminhamento & $11(4,03)$ \\
\hline Não informado & $146(53,48)$ \\
\hline
\end{tabular}

Fonte: elaboração própria. 
Conforme a Tabela 3, evidenciou-se maior proporção de vítimas do sexo feminino nos casos de violência física, psicológica, financeira e abandono/negligência, e maior proporção de vítimas do sexo masculino nos casos de autoabandono e violência institucional ( $p=0,017)$. Quanto à relação da vítima com o denunciante, observou-se maior proporção de denúncias de violência física, psicológica, financeira, por abandono/ negligência e institucional realizadas pelo próprio idoso, cônjuge ou filho, enquanto os casos de autoabandono foram denunciados, na maioria da vezes, por não familiares da vítima $(p<0,001)$. No que diz respeito à relação da vítima com 0 agressor, evidenciou-se que a maior proporção de casos de violência psicológica, financeira e por abandono/negligência foi cometida por cônjuges ou filhos, enquanto a maior proporção de casos de violência física e institucional foi cometida por não familiares $(p<0,001)$.

A análise de tendência por regressão linear simples foi significativa, o que evidenciou uma tendência ascendente de casos de violência contra idosos. 0 modelo de regressão estimado foi y $=21,4+3,7 \times\left(r^{2}=76 \%\right)$, 0 que significa que há um acréscimo médio de 3,7 denúncias ao ano, e a média do período foi de 21,4 casos. 0 Gráfico 1 mostra que houve uma tendência crescente de denúncias de violência entre os anos de 2004 e 2016.

Tabela 3. Distribuição de idosos segundo categoria de violência e características da vítima, do denunciante e do agressor (Alfenas, 2016)

\begin{tabular}{|c|c|c|c|c|c|c|c|c|}
\hline $\begin{array}{c}\text { Variáveis* } \\
\text { n(\%) }\end{array}$ & $\begin{array}{l}\text { Física } \\
\text { n(\%) }\end{array}$ & $\begin{array}{c}\text { Psicológica } \\
\text { n(\%) }\end{array}$ & $\begin{array}{c}\text { Financeira } \\
n(\%)\end{array}$ & $\begin{array}{c}\text { Abandono/ } \\
\text { negligência } \\
\text { n(\%) }\end{array}$ & $\begin{array}{c}\text { Autoaban- } \\
\text { dono } \\
\mathrm{n}(\%)\end{array}$ & $\begin{array}{c}\text { Institucional } \\
\mathrm{n}(\%)\end{array}$ & $\begin{array}{c}\text { Outras } \\
\text { n(\%) }\end{array}$ & $\mathbf{p}^{* *}$ \\
\hline \multicolumn{9}{|c|}{ Sexo da vítima } \\
\hline Feminino & $9(64,3)$ & $31(70,4)$ & $14(58,3)$ & $56(58,9)$ & $5(23,8)$ & $1(25,0)$ & $43(62,3)$ & 0,016 \\
\hline Masculino & $5(35,7)$ & $13(29,6)$ & $10(41,7)$ & $39(41,1)$ & $16(76,2)$ & $3(75,0)$ & $26(37,7)$ & \\
\hline \multicolumn{9}{|c|}{ Sexo do denunciante } \\
\hline Feminino & $7(63,6)$ & $26(61,9)$ & $11(73,3)$ & $60(75,0)$ & $11(64,7)$ & $2(50,0)$ & $41(71,9)$ & 0,676 \\
\hline Masculino & $4(36,4)$ & $16(38,1)$ & $4(26,7)$ & $20(25,0)$ & $6(35,3)$ & $2(50,0)$ & $16(28,1)$ & \\
\hline \multicolumn{9}{|c|}{ Relação da vítima com o denunciante } \\
\hline Próprio idoso & $4(36,4)$ & $18(47,4)$ & $4(33,3)$ & $9(15,8)$ & $1(7,1)$ & $3(52,6)$ & $20(33,9)$ & $<0,001$ \\
\hline Cônjuge/filho & $4(36,4)$ & $16(42,1)$ & $5(41,7)$ & $29(50,9)$ & $5(35,7)$ & $0(28,9)$ & $11(40,2)$ & \\
\hline Outros & $3(27,3)$ & $4(10,5)$ & $3(25,0)$ & $19(33,3)$ & $8(57,2)$ & $1(18,4)$ & $7(25,9)$ & \\
\hline \multicolumn{9}{|c|}{ Sexo do agressor } \\
\hline Feminino & $7(53,8)$ & $17(41,5)$ & $7(38,9)$ & $19(46,3)$ & $2(16,7)$ & $0(0,0)$ & $25(42,4)$ & 0,551 \\
\hline Masculino & $6(46,2)$ & $24(58,5)$ & $11(61,1)$ & $22(53,7)$ & $10(83,3)$ & $1(100,0)$ & $34(57,6)$ & \\
\hline \multicolumn{9}{|c|}{ Relação da vítima com o agressor } \\
\hline Cônjuge/filho & $6(46,2)$ & $20(51,3)$ & $9(56,2)$ & $53(77,9)$ & $2(12,5)$ & $1(33,3)$ & $35(55,6)$ & $<0,001$ \\
\hline Outros & $7(53,8)$ & $19(48,7)$ & $7(43,8)$ & $15(22,1)$ & $14(87,5)$ & $2(66,7)$ & $28(44,4)$ & \\
\hline
\end{tabular}

*Categoria "não informado" omitida. A diferença nos totais se deve à ausência de informação. $\star \star$ Valor de $p$ obtido por meio do Teste Exato de Fisher.

Fonte: elaboração própria. 
Gráfico 1. Tendência ajustada de casos de violência (Alfenas-MG, 2004-2016)

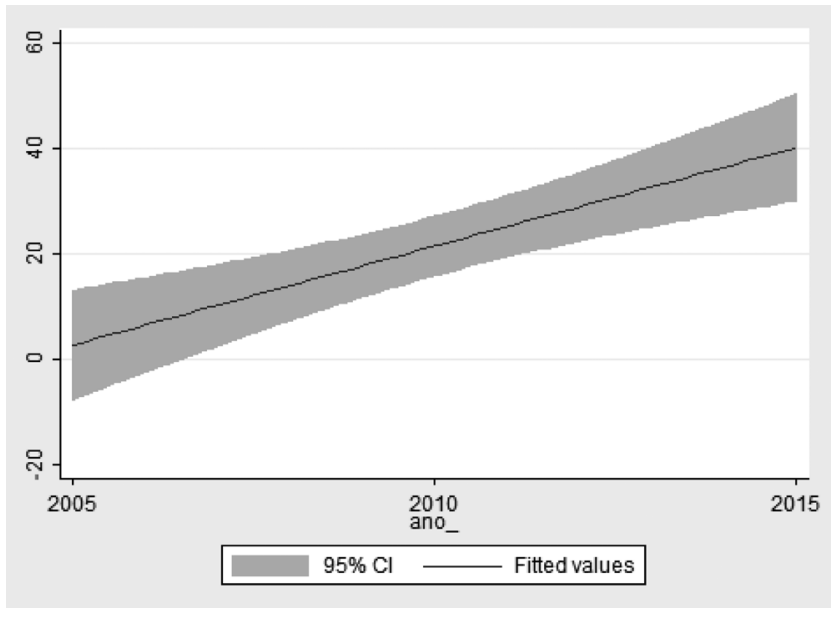

Fonte: elaboração própria.

\section{Discussão}

0 presente estudo analisou a violência praticada contra a pessoa idosa, segundo dados do Conselho Municipal do Idoso de um município do sul de Minas Gerais, Brasil. Quanto à caracterização da vítima, verificou-se que as mulheres foram vítimas de violência com maior frequência. Esse diferencial segundo o sexo, também observado por outros autores (7-9), mostra a relação de gênero, marcada pela assimetria e pela hierarquia nas relações, e revela uma sociedade machista, bem como uma cultura de discriminação contra a mulher $(1,10,11)$.

Este estudo apontou que idosos na segunda e terceira décadas do envelhecimento foram mais envolvidos em situação de violência, o que está em consonância com o estudo documental realizado em Itajaí, Santa Catarina, Brasil, o qual avaliou 72 denúncias do ano de 2012 no Creas (12). Outro estudo evidenciou que as maiores vítimas são os idosos mais velhos com limitações funcionais e cognitivas devido ao aumento do grau de dependência (11).

Com relação à situação conjugal das vítimas, houve predominância de idosos casados vítimas de violência, corroborando estudo realizado no Distrito Federal (13), Brasil, no qual idosos casados eram maioria - 43,04 \% das ocorrências; deve-se associar a isso as especificidades do companheiro e os achados que
0 apontam como segundo maior agressor (14). Entretanto, outras pesquisas evidenciaram maior prevalência de violência em idosos sem companhia marital, ou seja, viúvo, solteiro ou separado (15). Esse achado pode relacionar-se ao fato de a ausência de um companheiro também ser apontada como fator potencialmente associado a situações de negligência em idosos (16).

No que diz respeito ao denunciante, percebeu-se que o sexo feminino permaneceu com o maior percentual, o que também foi encontrado em estudo realizado em Porto Alegre, Brasil (8). Ainda, os mesmos autores destacam que há poucos estudos que descrevem informações sobre os denunciantes.

Quanto à faixa etária, mais uma vez 0 item não informado apresenta alto percentual, o que demonstra falhas no registro da denúncia. Admite-se que a incidência de denunciantes acima de 60 anos relaciona-se com os casos em que a própria vítima é a delatora, disponibilizando assim a informação sobre a idade. Além disso, tal situação está em consonância com a noção de envelhecimento ativo que está associado com a participação e com a autonomia (17). A noção de envelhecimento ativo considera que essa fase deve ser concebida como etapa da vida potencializadora de novas oportunidades, independentemente se existem situações de dependência ou fragilidade social (17), o que permite o reconhecimento da aptidão para alcançar a qualidade de vida satisfatória.

Os familiares foram os maiores responsáveis pelas denúncias dos casos de violência, indicando o papel familiar no amparo do idoso e na defesa de sua dignidade. A família e o Estado têm como atribuição o dever de cuidar do idoso e assegurar sua integridade física, moral e psicológica, segundo 0 artigo 230 da Constituição Federal (18).

Embora a família seja a principal rede de apoio, são seus próprios integrantes que, comumente, praticam a violência (19). Quanto ao grau de parentesco, observou-se, assim como em outros estudos $(7,13,14)$, que os filhos são os principais agressores. Um estudo que investigou a motivação da violência sob a perspectiva do agressor constatou que tal fato pode associar-se a esses indivíduos serem procedentes de lares violentos; assim, os filhos têm atos violentos contra os pais idosos porque foram criados à base de violência (20). Além disso, a dificuldade de um agressor em suprir suas próprias necessidades de vida pode fazer com que ele direcione ao idoso sua insatisfação em forma de violência (21). Observou-se, neste estudo, que os homens demostra- 
ram ser mais violentos; esse achado é compatível com o notado por outros pesquisadores $(8,14,20)$. Esse comportamento pode ser explicado pelo modelo social dominador e de supremacia masculina existente (22).

No presente estudo, analisaram-se os requerimentos de denúncia recebidos no Conselho Municipal do Idoso. Este tem como uma de suas atribuições receber e direcionar aos órgãos competentes as petições, as denúncias e as queixas sobre ameaças e descumprimento dos direitos da pessoa idosa, além de exigir das instâncias competentes as medidas cabíveis (23). Entretanto, uma porção significativa dos requerimentos identificados como denúncia de violência contra a pessoa idosa não se adequava à respectiva categoria. Tal situação pode ser um indicativo de que os profissionais que realizam os registros não estão qualificados para o reconhecimento da violência e para o direcionamento adequado em resposta às diversas demandas expostas pelos cidadãos que procuram o Conselho Municipal do Idoso.

0 elevado percentual de itens não informados na presente pesquisa, principalmente na categoria escolaridade, não permitiu a análise adequada de algumas variáveis. Assim como ocorre em outros estudos sobre violência, há carência de dados nos requerimentos de denúncia. Isso pode ser explicado por vários aspectos, a saber: ausência de instrumentos de registro padronizados, preenchimento incompleto das informações, dificuldade do idoso em relatar a ocorrência ou até mesmo de se deslocar até o local de denúncia, medo de ser repreendido pelo agressor (1) e falta de treinamento e capacitação do profissional que acolhe a denúncia.

Estudo documental realizado em Aracaju, Brasil (24), destacou a dificuldade de realizar pesquisas que desenvolvessem essa temática devido às fontes de dados se mostrarem escassas. Compatível com tal afirmação, foi observada, neste estudo, ausência de registros nos anos de 2005 e 2006. Nesse sentido, o uso da tecnologia e de sistemas de informação de apoio à gestão sobressaem como uma estratégia para as organizações no sentido de auxiliá-las no cumprimento de seus objetivos, garantindo agilidade, confiabilidade e qualidade de informações (25).

Neste estudo, verificou-se um aumento gradativo de denúncias de violências. Concomitantemente, no cenário nacional, notam-se as transformações na política de assistência ao idoso, que certamente podem influenciar no padrão de violência registrado. Dentre os dispositivos legais de amparo à pessoa idosa, destaca-se a implementação do Estatuto do Idoso, que é um instrumento de proteção ao direito dos idosos com previsão de pena pelo seu descumprimento (13).

Com relação ao desfecho das denúncias por parte do ConseIho Municipal do Idoso, a maioria dos casos de violência foi encaminhada ao Cras e ao Creas. Tais unidades compõem a rede de proteção social e visam, respectivamente, à prevenção da ocorrência de situações de vulnerabilidade social e ao fortalecimento de vínculo familiar e comunitário, bem como ao trabalho social com as famílias e indivíduos em situação de risco pessoal e social por violação de direitos (26).

Uma metanálise (27) sobre a prevalência de violência em idosos encontrou inicialmente 38.444 estudos sobre a temática e identificou a negligência, violência psicológica e financeira como as principais cometidas contra essa população, o que é compatível com os achados deste estudo e de outros na literatura nacional $(12,28)$ e internacional $(29)$.

A negligência é presente tanto em nível doméstico quanto em institucional no Brasil; dela sobrevêm, recorrentemente, lesões e traumas físicos, emocionais e sociais para a pessoa (30). A violência psicológica pode ter como consequências uma baixa autoestima, decréscimo da confiança e maior debilidade da vítima; pode gerar medo e dificuldades em tomar decisões e diminuir a dignidade individual. Como forma de violência financeira, pode-se encontrar a imposição de barreiras ao idoso de usar seus recursos em benefício próprio e a apropriação indevida dos bens do idoso, como, por exemplo, o uso do seu cartão de aposentadoria (7).

Ao evidenciar que mulheres idosas foram mais frequentemente vítimas de violência física, psicológica, financeira e abandono/ negligência, denota-se um fenômeno persistente e de variadas conformações que expressam a associação do feminino com a fragilidade e a passividade (31).

Ainda, no que se refere à questão de gênero, uma das atribuições historicamente conferidas ao homem é a de papel de chefe de família, provedor e detentor do poder (31). Essa construção da masculinidade e da associação da figura feminina aos cuidados de saúde pode culminar comportamentos que ameaçam a saúde do homem (32). Esse fato pode explicar a maior proporção de vítimas do sexo masculino nos casos de autoabandono evidenciada no presente estudo. 
Nesta pesquisa, observou-se que pessoas não familiares das vítimas denunciaram na maioria das vezes casos de autoabandono, diminuindo a frequência de denúncias em outros tipos de violência que, consequentemente, envolviam outros agressores que não a própria vítima. Assim, observa-se a necessidade de suscitar um processo consistente de informações sobre os direitos dos idosos e estimular a sociedade a comprometer-se efetivamente em prevenir, denunciar e enfrentar a violência contra o idoso (5).

No âmbito da saúde coletiva, os profissionais, em especial os enfermeiros, em virtude de sua proximidade com a população em questão, possuem um papel relevante na divulgação e discussão da violência na comunidade. Cabe destacar que toda visita da pessoa idosa à unidade de saúde deve ser entendida como uma oportunidade de investigar situações de violência. Assim, enfermeiros devem utilizar de estratégias de reconhecimento e enfrentamento do problema empregando instrumentos validados, dinâmicas em grupo e uma escuta atenta e qualificada durante a consulta de enfermagem (33).

Embora se configure como um grave problema de saúde pública, a violência contra a pessoa idosa ainda constitui uma questão velada na sociedade. Diante dessa realidade, é premente a implementação de uma rede articulada e contínua de ações, com definições claras do papel de cada membro, instituição, órgão governamental, setores da sociedade civil e profissionais no atendimento e prevenção da violência. Ademais, ressalta-se a necessidade de capacitação de profissionais que atuam nos serviços de saúde para agirem com competência na prevenção, identificação, enfrentamento e acompanhamento de situações de violência contra o idoso $(34,35)$.

A violência está estreitamente relacionada com o processo de envelhecimento-adoecimento. Assim, é necessário criar uma cultura que compreenda o processo de envelhecimento como uma etapa normal e irreversível da existência humana, na qual os idosos tenham a liberdade de viver com dignidade, respeito e com oportunidades de participação plena da vida social e sem violência $(34,35)$.
Cabe destacar que este estudo apresenta como limitação 0 registro incompleto dos requerimentos de denúncias. Ademais, o formulário de preenchimento das denúncias utilizado no Conselho Municipal de Saúde em questão não permite a inserção de informações sobre as condições de saúde do idoso e de sua funcionalidade, bem como de outros elementos relevantes sobre 0 agressor, as quais são fundamentais para a compreensão dos principais aspectos que envolvem a violência contra a pessoa idosa. Esse fato, aliado à falta de capacitação do profissional que registra a denúncia, dificulta a análise mais detalhada desse problema de saúde pública.

\section{Conclusões}

A violência constitui um fator de risco para problemas sociais e de saúde, entretanto é evitável e de responsabilidade da esfera social e da justiça. De acordo com o presente estudo, a violência contra o idoso mais prevalente foi a negligência, sobretudo contra as mulheres com idade entre 70 e 79 anos, cometida por agressor do sexo masculino e membro da própria família.

Os resultados encontrados neste estudo evidenciam a importância da capacitação dos profissionais que recolhem as denúncias bem como sobre a elaboração de uma ferramenta completa e padronizada para os registros de ocorrências.

Considerando que as várias expressões de violência podem ser prevenidas e reduzidas a partir do conhecimento dos diferentes aspectos que permeiam a violência contra idosos, sugere-se a ampliação de investigações na área, não somente documentais, mas também de pesquisas de campo, junto aos idosos da comunidade, a fim de preencher as lacunas no conhecimento sobre a extensão do problema, a eficácia dos programas de prevenção e 0 acesso aos serviços de apoio para 0 atendimento às vítimas de violência.

Conflito de interesse: nenhum declarado. 


\section{Referências}

1. Rodrigues RAP, Monteiro EA, Santos AMR, Ponte MLF, Fhon JRS, Bolina AF et al. Older adults abuse in three Brazilian cities. Rev Bras Enferm. 2017;70(4): 783-91. DOI: 10.1590/0034-7167-2017-0114

2. World Health Organization (WHO). Global status report on violence prevention. Geneva: WHO [citado em 20 abril 2018 ]. Disponível em: http://www.who.int/iris/handle/10665/145086

3. Correia TMP, Leal MCC, Marques APO, Salgado RAG, Melo HMA. Perfil dos idosos em situação de violência atendidos em serviço de emergência em Recife-PE. Rev Bras Geriatr Gerontol. 2012; 15(3): 529-36. DOI: 10.1590/S180998232012000300013

4. Bellal J, Mazhar K, Bardiya Z, Narong K, Tahereh O, Viraj P et al. Prevalence of Domestic Violence Among Trauma Patients. JAMA Surg. 2015; 150(12): 1177-83. DOI: 10.1001/jamasurg.2015.2386

5. Brasil. Subsecretaria Especial dos Direitos Humanos. Plano de ação para o enfrentamento da violência contra a pessoa idosa. Brasília: Subsecretaria Especial dos Direitos Humanos; 2007.

6. Cooper C, Livingston G. Intervening to reduce elder abuse: challenges for research. Age And Ageing. 2016; 45(2): 184-5. DOI: 10.1093/ageing/afw007.

7. Wanderbroocke ACNS, Moré CLOO. Estrutura e funcionamento familiar e a violência contra idosos. Psicol Argumento. 2013; 74(31): 395-403. DOI: 10.7213/psicolargum.v31i74.19929.

8. Crippa A, Rohde KLC, Schwanke CHA, Feijó AGS. Violência contra pessoa idosa a partir da análise de boletins de ocorrência. Sistema Penal \& Violência. 2016; 8(2): 220-30. DOI: 10.15448/2177-6784.2015.1.20576

9. Sampaio LS, Ferraira MJSF, Sampaio TSO, Souza WP, Prado APS, Reis LA. Violência Física em Idosos. C\&d - Rev Eletr Fainor. 2017; 2(10): 188-200. Disponível em: http://srv02.fainor.com.br/revista/index.php/memorias/article/view/661

10. Dias I. Violência doméstica e justiça: respostas e desafios. Sociologia: rev sociologia FLUP. 2010; 1(20): 245-62. Disponível em: http://ler.letras.up.pt/uploads/ficheiros/8796.pdf

11. Duque AM, Leal MCC, Marques APO, Eskinazi FMV, Duque AM. Violência contra idosos no ambiente doméstico: prevalência e fatores associados (Recife/PE). Ciênc Saúde Coletiva. 2012; 17(8): 2199-208. DOI: 10.1590/S1413-81232012000800030

12. Ploner KS, Hoffmann RM, Baldissera FB. Violência contra idosos: análise das denúncias e seu atendimento no Creas. RBCEH. 2015; 11(2): 141-51. DOI: 10.5335/rbceh.2012.4009

13. Oliveira MLC, Gomes ACG, Amaral CPM, Santos LB. Características dos idosos vítimas de violência doméstica no Distrito Federal. Rev Bras Geriatr Gerontol. 2012; 15(3): 555-66. DOI: 10.1590/S1809-98232012000300016

14. Irigaray TQ, Esteves CS, Pacheco JTB, Grassi-Oliveira R, Argimon IIL. Maus-tratos contra idosos em Porto Alegre, Rio Grande do Sul: um estudo documental. Estud. Psicol. 2016; 33(3): 543-51. DOI: 10.1590/1982-02752016000300017

15. Mascarenhas MDM, Andrade SSCA, Neves ACM, Pedrosa AAG, Silva MMA, Malta DC. Violência contra a pessoa idosa: análise das notificações realizadas no setor saúde - Brasil, 2010. Ciênc Saúde Coletiva. 2012; 17(9): 2331-41. DOI: $10.1590 /$ S1413-81232012000900014

16. Queiroz ZPV, Lemos NFD, Ramos LR. Fatores potencialmente associados à negligência doméstica entre idosos atendidos em programa de assistência domiciliar. Ciênc Saúde Coletiva. 2010; 15(6): 2815-24. DOI: 10.1590/S141381232010000600019

17. Carvalho MI. Cartografia das Políticas de Combate à Violência em Idosos. Um Estudo Exploratório. RASP. 2013; 1(1): 5476. Disponível em: https://www.researchgate.net/publication/277967007_Cartografia_das_Politicas_de_Combate_a_ Violencia_em_Idosos_Um_Estudo_Exploratorio

18. Brasil. Constituição da República Federativa do Brasil. Brasília, DF: Senado Federal; 1988.

19. Ribot VC, Rousseaux E, Garcia TC, Arteaga E, Ramos H, Alfonso M. Psychological the most common elder abuse in a Havana neighborhood. MEDICC Rev. 2017; 2(17): 39-43. Disponível em: http://www.medigraphic.com/pdfs/medicreview/ mrw-2015/mrw152i.pdf 
20. Silva CFS, Dias CMSB. Violência contra idosos: perfil sociodemográfico dos familiares agressores, tipos de violência impetrada e motivações para sua ocorrência. Rev Eletron Gestão Saúde. 2015;7(2):563-81. DOI: 10.18673/gs.v7i2.22040

21. Menezes MR. Violência contra idosos: é preciso se importar! Em: Berzins, MV, Malagutti, W. organizadores. Rompendo o silêncio: faces da violência na velhice. São Paulo: Matinari; 2010. p. 27-58.

22. Souza MB, Silva MS, Abreu GS. Violência Doméstica entre Parceiros Íntimos: Questões Culturais e Sociais acerca dos Homens Autores de Violência. Id Online: Rev Multidisc Psicol. 2017; 38(11): 388-407. DOI: 10.14295/idonline.v11i38.897

23. Brasil. Secretaria dos Direitos Humanos. Quer um conselho? Guia prático para a criação de conselhos e fundos estaduais e municipais de defesa dos direitos da pessoa idosa. Brasília: Secretaria dos Direitos Humanos; 2013.

24. Jesus JCL, Neves PSC. A violência contra idosos em Aracaju: um reflexo das modificações sociais da imagem de velhos em sociedades modernas 2010. Aracaju. Dissertação (mestrado em Sociologia) - Universidade Federal de Sergipe; 2010.

25. Mota TB, Oliveira Júnior AMC, Freitas AF. Desenvolvimento e uso de um software de gestão sob a ótica das dimensões organizacional, tecnológica e humana em empresas públicas. Navus: Rev Gestão Tecnol. 2016; 3(6): 70-87. Disponível em: https://dialnet.unirioja.es/servlet/articulo?codigo $=5580761$

26. Brasil. Conselho Nacional de Assistência Social (CNAS). Resolução n. ${ }^{\circ} 109$, de 11 de novembro de 2009. Aprova a Tipificação Nacional de Serviços Socioassistenciais. Diário Oficial da União 2009; 11 de novembro.

27. Yon Y, Mikton CR, Gassoumis ZD, Wilber KH. Elder abuse prevalence in community settings: a systematic review and meta-analysis. Lancet Glob Health. 2017; 5(2): 147-56. DOI: 10.1016/S2214-109X(17)30006-2

28. Santos AJ, Nicolau R, Fernandes AA, Gil AP. Prevalência da violência contra as pessoas idosas: uma revisão crítica da literatura. RCAAP. 2013; 1(72): 53-77. Disponível em: http://journals.openedition.org/spp/1192

29. Sooryanarayana R, Choo WY, Hairi NN, Chinna K, Hairi F, Ali ZM et al. The prevalence and correlates of elder abuse and neglect in a rural community of Negeri Sembilan state: baseline findings from The Malaysian Elder Mistreatment Project (MAESTRO), a population-based survey. BMJ Open. 2017;7(8):1-10.

30. Minayo MCS. Violência contra idosos: importante para um velho problema. Cad Saúde Pública. 20031 Bandeira L. Violência de gênero: a construção de um campo teórico e de investigação. Sociedade Estado. 2014; 29(2): 449-69.

31. Bandeira L. Violência de gênero: a construção de um campo teórico e de investigação. Sociedade Estado. 2014; 29(2): 449-69. DOI: 10.1590/S0102-69922014000200008

32. Machin R, Couto MT, Silva GSN, Schraiber LB, Gomes R, Santos F et al. Concepções de gênero, masculinidade e cuidados em saúde: estudo com profissionais de saúde da atenção primária. Ciênc Saúde Coletiva. 2011; 16(1): 4503-12. DOI: $10.1590 /$ S1413-81232011001200023

33. Castro VC, Rissardo LK, Carreira L. Violence against the Brazilian elderlies: an analysis of hospitalizations. Rev Bras Enferm. 2018; 71(suppl 2): 777-85. DOI: 10.1590/0034-7167-2017-0139

34. Alencar KCA, Santos JO, Hino P. Vivência de situação de violência contra idosos. Rev Enferm Atenção Saúde. 2014; 3(1): 74-83. Disponível em: http://seer.uftm.edu.br/revistaeletronica/index.php/enfer/article/view/932

35. Guimarães APS, Górios C, Rodrigues CL, Armond JE. Notificação de violência intrafamilar contra a mulher idosa na cidade de São Paulo. Rev Bras Geriatr Gerontol. 2018; 21(1): 91-7. DOI: 10.1590/1981-22562018021.160213 